\title{
REGULAR-EQUIVALENCE OF 2-KNOT DIAGRAMS AND SPHERE EVERSIONS
}

\author{
MASAMICHI TAKASE AND KOKORO TANAKA
}

Dedicated to Professor Yukio Matsumoto on the occasion of his seventieth birthday

\begin{abstract}
For each diagram $D$ of a 2 -knot, we provide a way to construct a new diagram $D^{\prime}$ of the same knot such that any sequence of Roseman moves between $D$ and $D^{\prime}$ necessarily involves branch points. The proof is done by developing the observation that no sphere eversion can be lifted to an isotopy in 4-space.
\end{abstract}

\section{INTRODUCTION}

A surface-knot (or a $\Sigma^{2}$-knot) is a submanifold of 4-space $\mathbb{R}^{4}$, homeomorphic to a closed connected oriented surface $\Sigma^{2}$. Two surface-knots are said to be equivalent if they can be deformed to each other through an isotopy of $\mathbb{R}^{4}$. In this paper we mainly study $S^{2}$-knots, also called 2 -knots, and especially their diagrams. A diagram of a surface-knot $F$ is the image of $F$ via a generic projection $\mathbb{R}^{4} \rightarrow \mathbb{R}^{3}$, that is equipped with a height information to distinguish the 4th coordinate. A diagram is composed of four kinds of local pictures shown in Figure 1, each of which depicts a neighborhood of a typical point - a regular point, a double point, an isolated triple point or an isolated branch point. Two surface-knot diagrams are said to be equivalent if they are related by (ambient isotopies of $\mathbb{R}^{3}$ and) a finite sequence of seven Roseman moves, shown in Figure 2, where we omit height information for simplicity. According to Roseman [21], two surface-knots are equivalent if and only if they have equivalent diagrams.

Since an oriented surface-knot has a trivial normal bundle, (after a suitable isotopy of $\left.\mathbb{R}^{4}\right)$ it has a diagram with no branch points $([3,6])$. In [23], such a "branch-free" diagram is called a regular diagram, and two regular diagrams are said to be regular-equivalent if they are related by a finite sequence of "branchfree" Roseman moves, that is, the moves of type $D 1, D 2, T 1$ and $T 2$ in Figure 2. Recently, many studies of surface-knot diagrams up to suitably restricted local deformations have been made $([10,13,20,23])$, since they are important in studying the (in)dependence among the seven Roseman moves (see e. g. [12]).

In [23], Satoh explicitly described a pair of regular diagrams of a $T^{2}$-knot which are mutually equivalent but not regular-equivalent, by computing certain elements of $H_{1}\left(T^{2} ; \mathbb{Z}\right)$ derived from the double point curves of regular diagrams. Note that his method turns out not to be effective for the case of $S^{2}$-knots, since $H_{1}\left(S^{2} ; \mathbb{Z}\right)=0$.

Date: February 23, 2018.

2000 Mathematics Subject Classification. Primary 57Q45, Secondary 57R40, 57R42.

Key words and phrases. 2-knot, sphere eversion, diagram, branch point, Roseman move, immersion, quadruple point.

The first-named author has been supported in part by Grant-in-Aid for Scientific Research (C), (No. 22540074), Japan Society for the Promotion of Science.

The second-named author thank J. Scott Carter for helpful comments on the draft. He has been supported in part by Grant-in-Aid for Scientific Research (C), (No. 26400082), Japan Society for the Promotion of Science. 


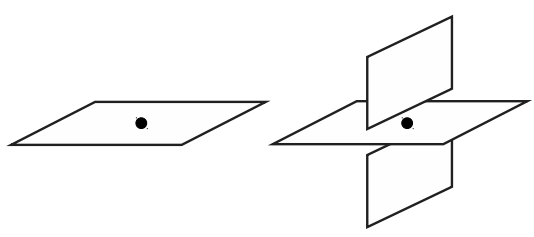

a regular point a double point

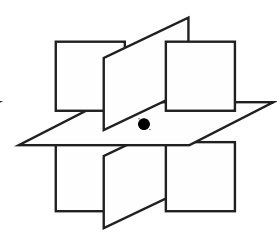

a triple point

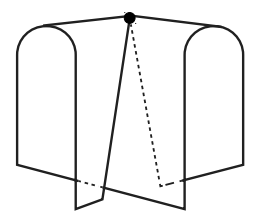

a branch point

FiguRE 1. Local pictures of the projection of a surface-knot
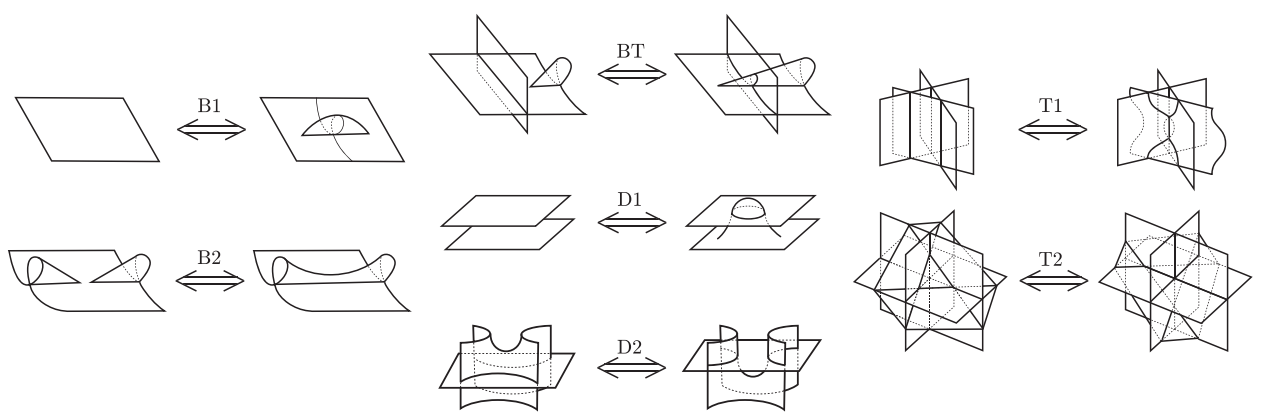

Figure 2. The Roseman moves

Recently, Oshiro and the second author proved Satoh's result by a different method using certain algebraic systems, called racks ([20, Section 6$])$. Note that it also turns out not to be effective for the case of $S^{2}$-knots (see [20, Section 6]).

In this paper, we present the first example with the same property in the case of oriented $S^{2}$-knots. Precisely, we provide a way to construct, given a regular diagram $D$ of an oriented $S^{2}$-knot, a new regular diagram $D^{\prime}$ such that $D$ and $D^{\prime}$ are mutually equivalent but not regular-equivalent (Theorem 3.6). Our method stands on basic material in immersion theory - especially on the number of quadruple points of a generic sphere eversion and that of generic immersions of 3-manifolds in 4-space, and is potentially useful for surface-knots other than $S^{2}$-knots. In the course of the argument, we observe that no sphere eversion can be lifted to an isotopy in $\mathbb{R}^{4}$ (Corollary 3.4).

Throughout the paper, we work in the smooth category; all manifolds, immersions and embeddings are supposed to be differentiable of class $C^{\infty}$, unless otherwise stated. We always deal with generic immersions and generic regular homotopies defined in a natural way (see [16]). We also suppose that the spheres are oriented.

We fix the following symbols.

- The orthogonal projection

$$
\begin{aligned}
& \pi: \quad \mathbb{R}^{n+1} \quad \longrightarrow \quad \mathbb{R}^{n}, \\
& \left(x_{1}, x_{2}, \ldots, x_{n+1}\right) \longmapsto\left(x_{1}, x_{2}, \ldots, x_{n}\right),
\end{aligned}
$$

defined by dropping off the last coordinate.

- The reflection

$$
\begin{aligned}
& r: \quad \mathbb{R}^{n} \quad \longrightarrow \quad \mathbb{R}^{n}, \\
& \left(x_{1}, x_{2}, \ldots, x_{n}\right) \longmapsto\left(-x_{1}, x_{2}, \ldots, x_{n}\right),
\end{aligned}
$$

obtained by multiplying the first coordinate by -1 .

- The standard inclusion $j: S^{2} \hookrightarrow \mathbb{R}^{3}$. 


\section{IMMERSION THEORY}

Smale's paradox states that one can turn a sphere inside out. More precisely, Smale proved that the standard inclusion $j: S^{2} \hookrightarrow \mathbb{R}^{3}$ and its reflection $j^{*}:=$ $r \circ j$ are regularly homotopic. Such a regular homotopy is called an eversion. Several examples of explicit eversions have been discovered and described by many mathematicians, including Arnold Shapiro and Bernard Morin [4] (see [1, §2] and [2] for detailed histories).

The following nature of a sphere eversion is well-known and plays a key role in this paper.

Proposition 2.1 (Max and Banchoff [16], see also [8]). Every sphere eversion has an odd number of quadruple points.

Proposition 2.1 states that in any regular homotopy between $j$ and $j^{*}$ an odd number of quadruple points appear. Nowik generalized this result for immersions of orientable surfaces as follows.

Let $f, f^{\prime}: \Sigma^{2} \rightarrow \mathbb{R}^{3}$ be two regularly homotopic generic immersions. Then Nowik [18] proved that the number modulo 2 of quadruple points occurring in generic a regular homotopy between $f$ and $f^{\prime}$ does not depend on the choice of the regular homotopy; we denote this invariant by $Q\left(f, f^{\prime}\right) \in \mathbb{Z} / 2 \mathbb{Z}$.

Theorem 2.2 (Nowik [19]). Let $f: \Sigma_{g}^{2} \rightarrow \mathbb{R}^{3}$ be a generic immersion of a closed orientable surface $\Sigma_{g}^{2}$ of genus $g$. Then, for an orientation-preserving diffeomorphism $\varphi$

$$
Q(f, f \circ \varphi)=\operatorname{rank}\left(\varphi_{*}-\mathrm{Id}\right) \quad(\bmod 2),
$$

and for an orientation-reversing diffeomorphism $\varphi$

$$
Q(f, f \circ \varphi)=\operatorname{rank}\left(\varphi_{*}-\mathrm{Id}\right)+g+1 \quad(\bmod 2),
$$

where $\varphi_{*}$ is the homomorphism on $H_{1}\left(\Sigma_{g}^{2} ; \mathbb{Z} / 2 \mathbb{Z}\right)$ induced by $\varphi$.

In particular when $\Sigma_{g}^{2}=S^{2}, Q(f, f \circ \varphi)$ equals 0 or $1(\bmod 2)$ depending on whether $\varphi$ is orientation-preserving or orientation-reversing.

Remark 2.3. For two generic immersions $f: \Sigma \rightarrow \mathbb{R}^{3}$ and $f^{\prime}: \Sigma^{\prime} \rightarrow \mathbb{R}^{3}$ of oriented surfaces, we can consider the oriented connected sum of $f$ and $f^{\prime}$ in the similar way to $[9$, p. 167] (see also $[14, \S 2]$ and [8, p. 502]). Roughly, the connected sum is an immersion of $\Sigma \sharp \Sigma^{\prime}$ whose image is formed by connecting the images of $f$ and $f^{\prime}$ by an untwisted tube in $\mathbb{R}^{3}$ with respect to the orientations. Note that the resultant immersion is subject to the choice of "the connecting tube", but two immersions constructed in this way are mutually regularly homotopic through $a$ regular homotopy with no quadruple points since we can move the tube keeping it free from the (isolated) triple points of $f$ and $f^{\prime}$.

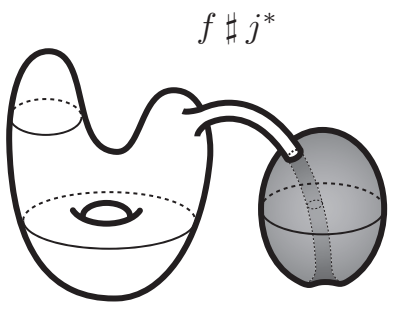

$$
f: \Sigma \rightarrow \mathbb{R}^{3} \quad j^{*}: S^{2} \rightarrow \mathbb{R}^{3}
$$

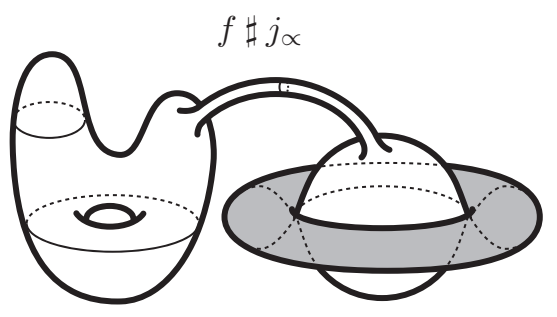

$$
f: \Sigma \rightarrow \mathbb{R}^{3}
$$$$
j_{\propto}: S^{2} \rightarrow \mathbb{R}^{3}
$$

Figure $3 . f \sharp j^{*}$ and $f \sharp j_{\propto}$

Now, by Nowik's result in [18], we can easily prove the following. 
Lemma 2.4. For a generic immersion $f: \Sigma \rightarrow \mathbb{R}^{3}$ of a closed orientable surface $\Sigma$,

$$
Q\left(f, f \sharp j^{*}\right)=1 \quad(\bmod 2) .
$$

Note that $f$ and $f \sharp j^{*}$ are regularly homotopic by [11, Theorem 1].

Proof. It is clear that in order to take the oriented connected sum $f \sharp j^{*}$ we need to yield new self-intersection points as in the left of Figure 3, so that we can consider $f \sharp j^{*}$ to be the connected sum $f \sharp j_{\propto}$ of $f$ and the immersion $j_{\propto}: S^{2} \rightarrow \mathbb{R}^{3}$ described in the right of Figure 3.

Since the immersion $j_{\propto}$ is the very first (or last) step in an explicitly described eversion (see [16, Fig. 2b, p. 199]). Therefore, we have an explicit regular homotopy between the standard inclusion $j$ and $j_{\propto}$, and hence between $f=f \sharp j$ and $f \sharp j \propto$, with a single quadruple point ([16, Fig. 14a, p. 206]). Hence the result follows from Nowik's result [18].

The following proposition, along with Lemma 2.4, will play a crucial role in the proof of our main theorem (Theorem 3.6).

Proposition 2.5. Let $f: S^{3} \rightarrow \mathbb{R}^{4}$ be a generic immersion of the 3 -sphere. If there exists an embedding $\tilde{f}: S^{3} \rightarrow \mathbb{R}^{5}$ such that $f=\pi \circ \tilde{f}$ for the orthogonal projection $\pi: \mathbb{R}^{5} \rightarrow \mathbb{R}^{4}$, then $f$ has an even number of quadruple points.

Proof. Let $\iota: \mathbb{R}^{4} \rightarrow \mathbb{R}^{5}$ be the inclusion. Then, $\iota \circ f$ is regularly homotopic to the embedding $\tilde{f}$. By [7], the Smale invariant of the embedding $\widetilde{f}$, considered as an element of $\pi_{3}(S O(5))$, lies in the kernel of the $J$-homomorphism

$$
J: \pi_{3}(S O(5)) \rightarrow \pi_{3}^{S} \approx \mathbb{Z} / 24 \mathbb{Z} .
$$

This implies that the cobordism class of the immersion $f$ is zero in the cobordism group of immersions of closed oriented 3 -manifolds in $\mathbb{R}^{4}$, which is known to be isomorphic to the stable 3 -stem $\pi_{3}^{S} \approx \mathbb{Z} / 24 \mathbb{Z}$. The reason is that to read off the immersion cobordism class of $f$, we just have to apply the Pontrjagin-Thom construction for $\iota \circ f\left(S^{3}\right) \subset \mathbb{R}^{5}$ and the natural normal framing derived from the codimension one immersion $f([5]$, see also [9, p. 180]), and this construction exactly gives the $J$-homomorphism.

Due to [5], the number modulo 2 of quadruple points of a generic immersion of a closed oriented 3 -manifold into $\mathbb{R}^{4}$ is invariant up to cobordism and determines the non-trivial homomorphism from $\pi_{3}^{S} \approx \mathbb{Z} / 24 \mathbb{Z}$ to $\mathbb{Z} / 2 \mathbb{Z}$. Thus, the null-cobordant immersion $f$ should have an even number of quadruple points.

Remark 2.6. Actually, for a generic immersion of $S^{3}$ into $\mathbb{R}^{4}$, its liftability to an embedding even in $\mathbb{R}^{6}$ implies that it has an even number of quadruple points (cf. [17, Example 1] and [24, Theorem 4.4]).

The following lemma is elementary.

Lemma 2.7. Let $\tilde{f}: S^{2} \rightarrow \mathbb{R}^{4}$ be an embedding of the 2 -sphere $S^{2}$ such that $f:=$ $\pi \circ \tilde{f}$ is an immersion. Then, there exists an embedding

$$
\widetilde{F}: D^{3} \rightarrow \mathbb{R}_{+}^{5}=\left\{\left(\left(x_{1}, x_{2}, x_{3}, x_{4}\right), t\right) \in \mathbb{R}^{4} \times[0, \infty)\right\}
$$

of the 3 -disk $D^{3}$ such that $\widetilde{F}$ is an embedding extending $\widetilde{f}$ and that $(\pi, \mathrm{Id}) \circ \widetilde{F}$ is an immersion extending $f$, where $(\pi, \mathrm{Id})$ is the projection

$$
(\pi, \mathrm{Id}): \mathbb{R}_{+}^{5} \rightarrow \mathbb{R}_{+}^{4}:\left(\left(x_{1}, x_{2}, x_{3}, x_{4}\right), t\right) \mapsto\left(\left(x_{1}, x_{2}, x_{3}\right), t\right) .
$$


Proof. By Kervaire's result [15], there exists a 3 -disk $\widetilde{\Delta}$ properly embedded in $\mathbb{R}_{+}^{5}$ such that $\partial \widetilde{\Delta}=\widetilde{f}\left(S^{2}\right)$. Then at each point $y \in \partial \widetilde{\Delta} \subset \mathbb{R}^{4}$, the vector $\left(\partial / \partial x_{4}\right)_{y}$ is not tangent to $\partial \widetilde{\Delta}$ since $f=\pi \circ \widetilde{f}$ is an immersion. Therefore, we can assume that $\partial / \partial x_{4}$ determines a normal vector field on $\partial \widetilde{\Delta}$ and extend it to a (homotopically unique) normal vector field on $\widetilde{\Delta}$ in $\mathbb{R}_{+}^{5}$, which we denote by $\nu$.

By the Compression Theorem [22], we can isotope $(\widetilde{\Delta}, \nu)$ with fixing it on $\partial \widetilde{\Delta}$ so that $\nu$ becomes parallel to the $x_{4}$-direction (of $\mathbb{R}_{+}^{5}$ ). Thus, we now have the embedded 3-disk $\widetilde{\Delta}$ in $\mathbb{R}_{+}^{5}$ with boundary $\partial \widetilde{\Delta}=\widetilde{f}\left(S^{2}\right) \subset \partial \mathbb{R}_{+}^{5}=\mathbb{R}^{4}$, whose projection via $\left(\pi\right.$, Id) is an immersed 3-disk in $\mathbb{R}_{+}^{4}$ with boundary $f\left(S^{2}\right) \subset \partial \mathbb{R}_{+}^{4}=\mathbb{R}^{3}$. Thus, we can have a desired embedding $\widetilde{F}: D^{3} \rightarrow \mathbb{R}_{+}^{5}$ such that $\widetilde{F}$ is an embedding extending $\widetilde{f}$ and $(\pi, \mathrm{Id}) \circ \widetilde{F}$ is an immersion extending $f$.

\section{Equivalent but not Regular-equivalent diagrams}

For an oriented surface-knot diagram $D$, the mirrored diagram of $D$ is defined to be $r(D)$ and the inverted diagram of $D$ be the same diagram as $D$ with the opposite orientation. We use the symbols $D^{*}$ for the mirrored diagram and $-D$ for the inverted diagram.

Remark 3.1. It is seen that the diagram $D$ equipped with the reversed height information represents the same surface-knot as the diagram $r(D)$. Note, however, that we always use the term mirrored diagram and the symbol $D^{*}$ exactly for $r(D)$.

Proposition 3.2. Let $D$ be a regular diagram of an $S^{2}$-knot.

(i) The diagram $D$ and its mirrored diagram $D^{*}$ are not regular-equivalent.

(ii) The diagram $D$ and its inverted diagram $-D$ are not regular-equivalent.

Proof. First, we prove the case (i). Assume that $D$ and $D^{*}(=r(D))$ are regularequivalent, that is, there is a sequence of Roseman moves without branch points. Clearly such a sequence of Roseman moves gives the regular homotopy covered by its corresponding isotopy. Namely there exists an isotopy

$$
\widetilde{h}_{t}: S^{2} \rightarrow \mathbb{R}^{4}, t \in[-1,1],
$$

whose projection gives a regular homotopy

$$
h_{t}\left(:=\pi \circ \widetilde{h}_{t}\right): S^{2} \rightarrow \mathbb{R}^{3}, t \in[-1,1]
$$

such that $h_{1}\left(S^{2}\right)=\pi \circ \widetilde{h}_{1}\left(S^{2}\right)=D$ and $h_{-1}\left(S^{2}\right)=\pi \circ \widetilde{h}_{-1}\left(S^{2}\right)=D^{*}$.

Then, we will show below that the above assumption leads to the following two contradictory claims regarding the number of quadruple points occurring in $h_{t}$ :

(a) $Q\left(h_{-1}, h_{1}\right)=1(\bmod 2)$, and

(b) $Q\left(h_{-1}, h_{1}\right)=0(\bmod 2)$.

Roughly speaking, (a) will be deduced from the fact that $h_{t}$ contains an essential part of an eversion, and (b) from the fact that $h_{t}$ lifts to the isotopy $\widetilde{h}_{t}$.

(a) We construct a regular homotopy $\left\{\phi_{t}\right\}_{t \in[-3,2]}$, which is an eversion, between $\phi_{2}=i$ and $\phi_{-3}=r \circ i$ as follows (see Figure 4). For $t \in[-1,1]$, we put $\phi_{t}=h_{t}$. Since all immersions of $S^{2}$ into $\mathbb{R}^{3}$ are regularly homotopic, there exists a regular homotopy between the immersion $h_{1}$ and the standard inclusion $i$; let $\left\{\phi_{t}\right\}_{t \in[1,2]}$ be such a regular homotopy that $\phi_{1}=h_{1}$ and $\phi_{2}=i$. Now, since the images of $h_{-1}$ and $r \circ h_{1}$ coincide (as oriented immersed surfaces), there exists an orientation-preserving diffeomorphism $\varphi$ of $S^{2}$ such that $r \circ h_{1}=h_{-1} \circ \varphi$. Then, take a regular homotopy $\left\{\phi_{t}\right\}_{t \in[-2,-1]}$ such that $h_{-1} \circ \varphi\left(=\phi_{-2}\right)$ and $h_{-1}\left(=\phi_{-1}\right)$. Finally, for $t \in[-3,-2]$ we put $\phi_{t}=r \circ \phi_{-1-t}$, so that $\phi_{-3}\left(=r \circ \phi_{2}\right)=r \circ i$ and $\phi_{-2}\left(=r \circ \phi_{1}\right)=r \circ h_{1}$. Note 


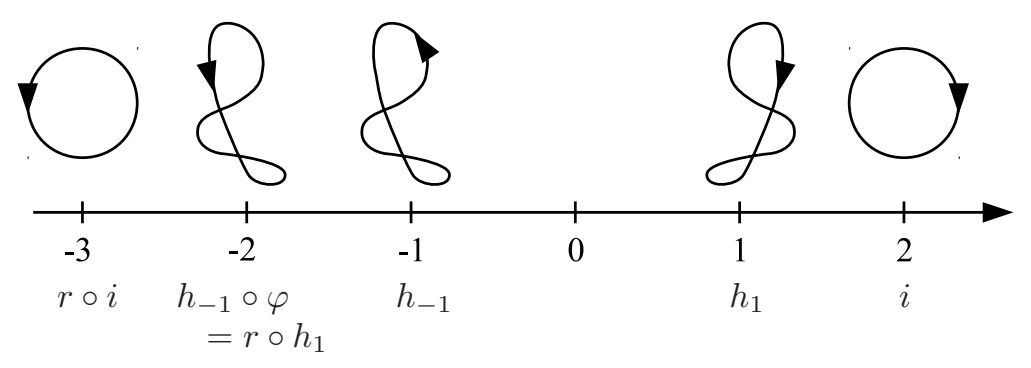

FiguRE 4. Regular homotopy $\left\{\phi_{t}\right\}_{t \in[-3,2]}$

that the number of quadruple points appearing in $\left\{\phi_{t}\right\}_{t \in[1,2]}$ and that appearing in $\left\{\phi_{t}\right\}_{t \in[-2,-3]}$ are the same.

Now it is clear that

$$
\begin{aligned}
Q\left(\phi_{-3}, \phi_{2}\right) & =Q\left(\phi_{-3}, \phi_{-2}\right)+Q\left(\phi_{-2}, \phi_{-1}\right)+Q\left(\phi_{-1}, \phi_{1}\right)+Q\left(\phi_{1}, \phi_{2}\right) \quad(\bmod 2), \\
& =Q\left(\phi_{-2}, \phi_{-1}\right)+Q\left(h_{-1}, h_{1}\right)+2 Q\left(\phi_{1}, \phi_{2}\right) \quad(\bmod 2) .
\end{aligned}
$$

Since we see that $Q\left(\phi_{-3}, \phi_{2}\right)=1(\bmod 2)$ by Proposition 2.1 and $Q\left(\phi_{-2}, \phi_{-1}\right)=0$ $(\bmod 2)$ by Theorem 2.2 , we have

$$
Q\left(h_{-1}, h_{1}\right)=1 \quad(\bmod 2) .
$$

(b) By Lemma 2.7, we can take an embedding

$$
\widetilde{F}: D^{3} \rightarrow \mathbb{R}_{\geq 1}^{5}=\mathbb{R}^{4} \times[1, \infty)
$$

such that $\widetilde{F}$ is an embedding extending $\widetilde{h}_{1}$ and that whose projection $F:=(\pi, \mathrm{Id}) \circ \widetilde{F}$ is an immersion extending $h_{1}$. Then, $(r,-$ Id $) \circ \widetilde{F}$, where

$$
\begin{aligned}
& (r,- \text { Id }): \quad \mathbb{R}^{n} \times \mathbb{R} \quad \longrightarrow \quad \mathbb{R}^{n} \times \mathbb{R}, \\
& \left(x_{1}, x_{2}, \ldots, x_{n}, t\right) \longmapsto\left(-x_{1}, x_{2}, \ldots, x_{n},-t\right),
\end{aligned}
$$

gives an embedding extending $\widetilde{h}_{-1}$ in $\mathbb{R}_{\leq-1}^{5}$, whose projection $(r,-$ Id $) \circ F$ is an immersion extending $h_{-1}$. Note that immersions $F$ and $(r,-\mathrm{Id}) \circ F$ have the same number of quadruple points.

Hence, by using $F,(r,-\mathrm{Id}) \circ F$ and $\left\{h_{t}\right\}_{t \in[-1,1]}$, we can construct an immersion of the 3-sphere $S^{3}=\left(-D^{3}\right) \cup\left(S^{2} \times[-1,1]\right) \cup D^{3}$ into $\mathbb{R}^{4}=\mathbb{R}^{3} \times \mathbb{R}$, which lifts to an embedding into $\mathbb{R}^{5}=\mathbb{R}^{4} \times \mathbb{R}$. Now, the number of quadruple points of this immersion, which is even by Proposition 2.5, is equal to the sum of the number of quadruple points in $\left\{h_{t}\right\}_{t \in[-1,1]}$ and twice that of $F$. Therefore, we have

$$
Q\left(h_{-1}, h_{1}\right)=0 \quad(\bmod 2) .
$$

This completes the proof by contradiction.

For the case (ii), the proof is almost the same. Take $h_{t}$ and $\widetilde{h}_{t}$ similarly as above. Then, (a) follows directly from Theorem 2.2, since $h_{-1}=h_{1} \circ \gamma$ for some orientation reversing diffeomorphism $\gamma$ of $S^{2}$. In order to show (b), we have only to consider $(\mathrm{Id},-\mathrm{Id}) \circ F$ instead of $(r,-\mathrm{Id}) \circ F$ in the above argument.

Let $F$ be an oriented surface-knot. We say that $F$ is $(+)$-amphicheiral if a diagram of $F$ is equivalent to its mirrored diagram and that $F$ is invertible if a diagram of $F$ is equivalent to its inverted diagram. Proposition 3.2 implies the following:

Theorem 3.3. Let $D$ be a regular diagram of an $S^{2}$-knot $K$. 
(i) If $K$ is a $(+)$-amphicheiral knot, then the two diagrams $D$ and $D^{*}$ of $K$ are equivalent but not regular-equivalent.

(ii) If $K$ is an invertible knot, then two diagrams $D$ and $-D$ of $K$ are equivalent but not regular-equivalent.

We have the following direct corollary.

Corollary 3.4. The trivial diagram of the trivial $S^{2}$-knot is not regular-equivalent to itself with the reversed orientation. In other words, we cannot lift a sphere eversion to any isotopy in $\mathbb{R}^{4}$.

It is natural to try to generalize Theorem 3.3 for other $S^{2}$-knot diagrams. In fact, we can prove in a similar way Theorem 3.6 below, which enables us to construct, from any given $S^{2}$-knot diagram $D$, a new diagram equivalent but not regularequivalent to $D$.

We first need the following definition.

Definition 3.5. Let $D_{0}$ be a regular diagram of the trivial $S^{2}$-knot which is obtained by spinning the tangle diagram $\succ$, and $y_{0} \in D_{0}$ be the regular point of $D_{0}$ as in Figure 5. For a surface-knot diagram $D$, choose a regular point $y$ of $D$ and take an oriented connected sum of $D$ and $D_{0}$ by introducing an untwisted tube connecting (disks around) $y$ and $y_{0}$ as in Figure 5 . Then the resulting diagram is called an everted diagram of $D$ and denoted by $D_{\propto}$. Although $D_{\propto}$ depends on the choices of $y$ and the connecting tube, but it is well-defined up to regular-equivalence.

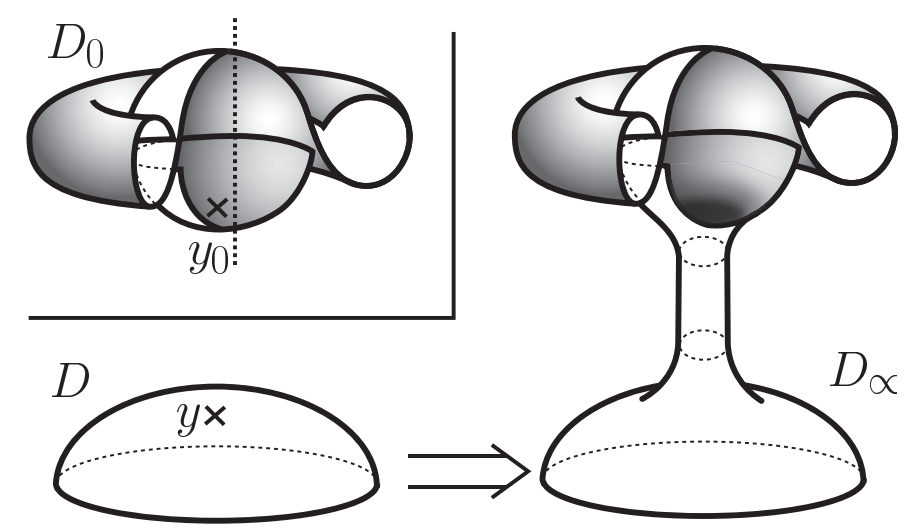

Figure 5. An everted diagram of $D$

Theorem 3.6. Let $D$ be a regular diagram of an $S^{2}$-knot $K$. Then, $D$ and its everted diagram $D_{\propto}$ are equivalent but not regular-equivalent.

Proof. As in the proof of Theorem 3.3, assume that $D$ and $D_{\propto}$ are regularequivalent, that is, assume the existence of an isotopy

$$
\widetilde{h}_{t}: S^{2} \rightarrow \mathbb{R}^{4}, t \in[0,1],
$$

whose projection gives a regular homotopy

$$
h_{t}: S^{2} \rightarrow \mathbb{R}^{3}, t \in[0,1]
$$

such that $h_{0}\left(S^{2}\right)=\pi \circ \widetilde{h}_{0}\left(S^{2}\right)=D_{\propto}$ and $h_{1}\left(S^{2}\right)=\pi \circ \widetilde{h}_{1}\left(S^{2}\right)=D$. From this assumption we will deduce the following two contradictory claims:

(a) $Q\left(h_{0}, h_{1}\right)=1(\bmod 2)$, and

(b) $Q\left(h_{0}, h_{1}\right)=0(\bmod 2)$. 
(a) Put $j^{*}=r \circ j: S^{2} \hookrightarrow \mathbb{R}^{3}$. Then, we can take the oriented connected sum $h_{1} \sharp j^{*}$ of $h_{1}$ and $j^{*}$ (as in Remark 2.3), so that $\left(h_{1} \sharp j^{*}\right)\left(S^{2}\right)=D_{\propto}$. Hence, there exists an orientation-preserving diffeomorphism $\varphi$ of $S^{2}$ such that

$$
h_{0}=\left(h_{1} \sharp j^{*}\right) \circ \varphi .
$$

Moreover, by Theorem 2.2, for any regular homotopy $\left\{h_{t}: S^{2} \rightarrow \mathbb{R}^{3}\right\}_{t \in[-1,0]}$ such that $h_{-1}=h_{1} \sharp j^{*}$ and $h_{0}=\left(h_{1} \sharp j^{*}\right) \circ \varphi$, we have

$$
Q\left(h_{-1}, h_{0}\right)=0 \quad(\bmod 2) .
$$

Since the whole regular homotopy $\left\{h_{t}\right\}_{t \in[-1,0] \cup[0,1]}$ between $h_{-1}=h_{1} \sharp j^{*}$ and $h_{1}$ has odd number of quadruple points by Lemma 2.4, we have

$$
Q\left(h_{0}, h_{1}\right)=1 \quad(\bmod 2) .
$$

(b) As seen above, we can consider $D_{\propto} \subset \mathbb{R}^{3} \times\{0\}$ as an oriented connected sum of $D=h_{1}\left(S^{2}\right)$ and $D_{0}$. Hence, there exists a cobordism $C$ between $D_{\propto}$ and the disjoint union $D \amalg D_{0}$, which is realized by attaching a 2 -handle along the "meridian" of the tube used in taking the connected sum. It is easy to see that the cobordism $C$ can be embedded in $\mathbb{R}^{4} \times[-1,0]$ whose projected image onto $\mathbb{R}^{3} \times[-1,0]$ is immersed and contains no quadruple points; assume that $C \subset \mathbb{R}^{4} \times[-1,0]$ such that $(\pi, \operatorname{Id})\left(C \cap\left(\mathbb{R}^{4} \times\{-1\}\right)\right)=D \amalg D_{0}$ and $(\pi, \operatorname{Id})\left(C \cap\left(\mathbb{R}^{4} \times\{0\}\right)\right)=D_{\propto}($ see Figure 6).

We can extend the embedding $\widetilde{h}_{1}$ to an embedding $\widetilde{F}: D^{3} \rightarrow \mathbb{R}_{>1}^{5}$ as in Lemma 2.7. It is clear that we can deform the diagram $D_{0}$ to the trivial diagram just by a single Roseman move of type $D 1$ (see Figure 2), that involves neither branch points nor quadruple points (in the projection onto $\mathbb{R}^{4}$ ). By capping off the trivial diagram with the embedded 3 -disk, we obtain an embedding $\widetilde{G}: D^{3} \rightarrow \mathbb{R}_{\leq-1}^{5}$ such that $(\pi, \operatorname{Id})\left(\widetilde{G}\left(D^{3}\right) \cap\left(\mathbb{R}^{4} \times\{-1\}\right)\right)=D_{0}$ and that the projected image of $\widetilde{G}\left(D^{3}\right)$ involves no quadruple points. Capping off the embedded cobordism $C$ with the embeddings $(\mathrm{Id},-\mathrm{Id}) \circ \widetilde{F}$ and $\widetilde{G}$, we obtain an embedded 3-disk $C^{\prime}$ in $\mathbb{R}_{-}^{5}$ such that $(\pi, \mathrm{Id})\left(C^{\prime} \cap\left(\mathbb{R}^{4} \times\{0\}\right)\right)=D_{\propto}$ (see Figure 6$)$. Note that the number of quadruple points of the projected image of $C^{\prime}$ is equal to that of $\widetilde{F}\left(D^{3}\right)$.

Hence, by using the track of the isotopy $\left\{h_{t}\right\}_{t \in[0,1]}$, the embedding $\widetilde{F}$, and the embedded 3-disk $C^{\prime}$, we can construct an immersion of $S^{3}$ into $\mathbb{R}^{4}$ covered by an embedding into $\mathbb{R}^{5}$ (see Figure 6).

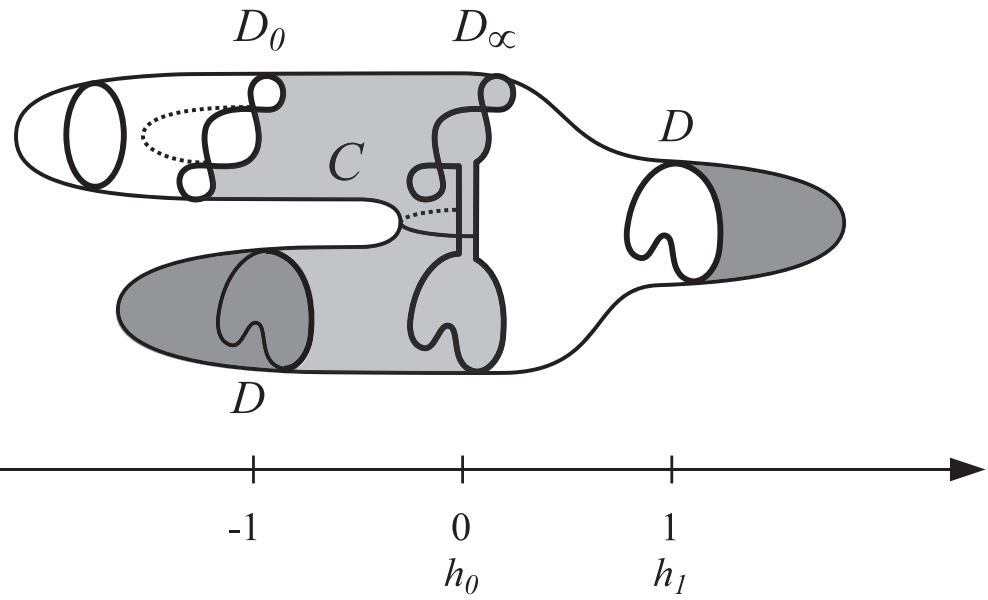

Figure 6. The immersed $S^{3}$ in $\mathbb{R}^{4}$ 
Now, the number of quadruple points of this immersion, which ought to be even by Proposition 2.5, has the same parity as the sum of $Q\left(h_{0}, h_{1}\right)$ and twice the number of quadruple points of the projected image of $\widetilde{F}\left(D^{3}\right)$. Therefore we have

$$
Q\left(h_{0}, h_{1}\right)=0 \quad(\bmod 2) .
$$

This completes the proof by contradiction.

Remark 3.7. For an oriented diagram $D$ of a $S^{2}$-knot with branch points, an arbitrarily chosen regular diagram $D^{\prime}$ (of the same knot) is not regular-equivalent to $D$, since it is obvious that any sequence of Roseman moves from $D^{\prime}$ to $D$ should contain a "branch-birth" move B1 or B2 shown in Figure 2.

\section{REFERENCES}

[1] Aitchison, Iain R.: The Holiverse: holistic eversion of the 2-sphere, preprint 2010, available at arXiv:1008.0916. 2

[2] Carter, J. Scott: An excursion in diagrammatic algebra: Turning a sphere from red to blue, Series on Knots and Everything, 48 (2012), World Sci. Publ. 2

[3] Carter, J. Scott; Saito, Masahico: Canceling branch points on projections of surfaces in 4-space, Proc. Amer. Math. Soc. 116 (1992), no. 1, 229-237. 1

[4] Francis, George K.; Morin, Bernard: Arnold Shapiro's eversion of the sphere, Math. Intelligencer 2 (1979/80), 200-203. 2

[5] Freedman, Michael H.: Quadruple points of 3-manifolds in $S^{4}$, Comment. Math. Helv. 53 (1978), 385-394. 2

[6] Hirsch, M. W.: Immersions of manifolds, Trans. Amer. Math. Soc. 93 (1959), 242-276. 1

[7] Hughes, John F.; Melvin, Paul M.: The Smale invariant of a knot, Comment. Math. Helv. 60 (1985), 615-627. 2

[8] Hughes, John F.: Another proof that every eversion of the sphere has a quadruple point, Amer. J. Math. 107 (1985), 501-505. 2.1, 2.3

[9] Hughes, John F.: Bordism and regular homotopy of low-dimensional immersions, Pacific J. of Math. 156 (1992), 155-184. 2.3, 2

[10] Jabłonowski, M.: Knotted surfaces and equivalencies of their diagrams without triple points, J. Knot Theory Ramifications 21 (2012), 1250019 (6 pages). 1

[11] Kaiser, Uwe: Immersions in codimension 1 up to regular homotopy, Arch. Math. (Basel) 51 (1988), 371-377. 2.4

[12] Kawamura, Kengo: On relationship between seven types of Roseman moves, to appear in Topology Appl. 1

[13] Kawamura, Kengo; Oshiro, Kanako; Tanaka, Kokoro: Independence of Roseman moves including triple points, in preparation. 1

[14] Kervaire, Michel A.: Sur l'invariant de Smale d'un plongement, (French) Comment. Math. Helv. 34 (1960), 127-139. 2.3

[15] Kervaire, Michel A.: Knot cobordism in codimension two, Manifolds-Amsterdam 1970 (Proc. Nuffic Summer School) pp. 83-105 Lecture Notes in Mathematics, Vol. 197 Springer, Berlin. 2

[16] Max, Nelson; Banchoff, Tom: Every sphere eversion has a quadruple point, Contributions to analysis and geometry (Baltimore, Md., 1980), pp. 191-209, Johns Hopkins Univ. Press, Baltimore, Md., 1981. 1, 2.1, 2

[17] Melikhov, S. A.: Sphere eversions and realization of mappings, Proc. Steklov Inst. Math. 247 (2004), 143-163. 2.6

[18] Nowik, Tahl: Quadruple points of regular homotopies of surfaces in 3-manifolds, Topology 39 (2000), 1069-1088. 2, 2, 2

[19] Nowik, Tahl: Automorphisms and embeddings of surfaces and quadruple points of regular homotopies, J. Differential Geom. 58 (2001), 421-455. 2.2

[20] Oshiro, Kanako; Tanaka, Kokoro: On rack colorings for surface-knot diagrams without branch points, to appear in Topology Appl. 1, 1

[21] Roseman, Dennis: Reidemeister-type moves for surfaces in four-dimensional space, Knot theory (Warsaw, 1995), 347-380, Banach Center Publ., 42, Polish Acad. Sci., Warsaw, 1998 1

[22] Rourke, Colin; Sanderson, Brian: The compression theorem I, Geom. Topol. 5 (2001), 399429. 2

[23] Satoh, Shin: Double decker sets of generic surfaces in 3-space as homology classes, Illinois J. Math. 45 (2001), 823-832. 1, 1 
[24] Takase, Masamichi: An Ekholm-Szücs-type formula for codimension one immersions of 3manifolds up to bordism, Bull. London Math. Soc. 32 (2007), 163-178. 2.6

Faculty of Science and Technology, Seikei University, 3-3-1 Kichijoji-kitamachi, Musashino, TOKYO 180-8633, JAPAN

E-mail address: mtakase@st.seikei.ac.jp

Department of Mathematics, Tokyo Gakugei University, 4-1-1 Nukuikita-machi, KoGANEI, TOKYO 184-8501, JAPAN

E-mail address: kotanaka@u-gakugei.ac.jp 